\title{
Bromocriptine alone or associated with L-dopa plus benserazide in Parkinson's disease
}

\author{
T.A. CAR A C E I, I. CELA NO, E. PAR A T I, A N D F. G I R O T T I \\ From the Istituto Neurologico 'C. Besta', Milan, Italy
}

S UMMARY Twenty-six patients affected by Parkinson's disease were treated with a 2-Br-alphaergocriptine (CB 154): 14 cases were given CB 154 alone, and 12 were given CB 154 along with L-dopa plus benserazide (Madopar). Both CB 154 and combined therapy (CB 154+Madopar) induced a significant improvement in total disability score, tremor, rigidity, akinesia, selfsufficiency, and some motor performance tests (dynamic tests). No significant difference was found between results obtained with CB 154 therapy and with Madopar treatment, while the improvement induced by combined therapy (CB 154+Madopar) was significantly higher than that obtained by Madopar alone. The adverse reactions caused by CB 154 alone or associated with Madopar are similar to those observed during other dopaminergic treatment. CB 154 alone or combined with Madopar appears to be a useful advance in the management of Parkinson disease.

2-bromo- $\alpha$-ergocriptine (CB 154) is an ergot alkaloid which has been proved effective in suppressing lactation and inhibiting prolactin secretion (Flückiger and Wagner, 1968; Billiter and Flückiger, 1971). As several experimental studies on animals (Corrodi et al., 1973; Miyamoto et al., 1974; Goldstein et al., 1975) showed that CB 154 has a dopamine stimulating effect both at presynaptic and postsynaptic levels, bromocriptine has been used in the treatment of Parkinson's disease (Calne et al., 1974; Lieberman et al., 1976a; Gerlach, 1976; Parkes et al., 1976; Gautier and Durand, 1977).

Another ergoline derivate (lergotrile), which reduces plasma prolactin concentration has been tried in the treatment of Parkinson's disease with not very satisfactory results (Lieberman et al., 1975). The results obtained with CB 154 in the treatment of Parkinson's disease are certainly valid although with different effects observed by different authors. According to Kartzinel et al. (1976a) CB 154 is more effective than L-dopa plus carbidopa (Sinemet), while Gerlach (1976) notes that CB 154 is less effective than L-dopa plus benserazide (Madopar). Lieberman et al. (1976b) claim that CB 154 would be useful in patients no

Address for correspondence and reprint requests: Professor Tommaso Caraceni, Istituto Neurologico 'C. Besta', Via Celoria 11, 20133 Milan, Italy.

Accepted 15 July 1977 longer responding to L-dopa. The addition $\stackrel{\vec{P}}{\mathrm{~N}}$ caffeine did not enhance the antiparkinsonisn potency of ergocriptine (Kartzinel et al., 1976b).

Taking this diversity of opinions regarding the therapeutic effectiveness of 2-bromo- $\alpha$-ergocriptine into account, it seemed interesting to compare the results obtained with the various therapies follows: (1) CB 154 versus no specific treatment; $\overrightarrow{0}$ (2) CB 154 versus Madopar; and (3) CB 154 plus $\searrow$ Madopar versus Madopar alone.

\section{Subjects and methods}

Twenty-six subjects (Table 1), 14 females and $\frac{2}{\triangleright}$ 12 males, with a mean age of 59.5 years (maxi- $\stackrel{2}{\overrightarrow{7}}$ mum 68 years and minimum 46 years) consented $\overrightarrow{0}$ to participate in this trial after being informed of 3 the possible risks and benefits. All the subjects were affected by Parkinson's disease; in three cases the disease was just beginning and sl'ght, seven patients were grade 2,12 were grade 3 , and 0 four were grade 4 (according to the Webster 욱 scale). The clinical pattern in most patients consisted of rigidity and akinesia; tremor was actually $\delta$ evident in only seven cases.

The mean duration of the illness was 5.4 years 음 (range five months to 13 years). The patients, $D$ with the exception of the three in the first stage of the disease, had been under treatment for a mean of three years (range six months to six years) o 
with various antiparkinsonism substances: L-dopa and decarboxylase inhibitor (Madopar) 18, Ldopa six; piribedil four; and benztropine six. One patient underwent stereotactic surgery (unilateral thalamotomy).

The on-off phenomenon was present in two patients, abnormal involuntary movements were present in two others, and hallucinations and dementia in one case. Before beginning the pharmacological trials the previous drug therapies (L-dopa, benztropine) were suspended and all patients, with the exception of the three without treatment, were given or continued Madopar therapy. Our patients were divided into two groups.

The first group consisted of 14 patients, of whom 11 received Madopar treatment (mean dosage of $625 \mathrm{mg}$ daily) and three, in the first stage of the disease, did not receive any antiparkinsonism therapy before entering the trial. The Madopar treatment was withdrawn for at least five days, and then the CB 154 treatment was begun in the same subjects. In all 14 patients treatment was begun with a daily dosage of $5 \mathrm{mg}$, increasing this by $5 \mathrm{mg}$ every third day until optimum efficacy was reached. The treatment was carried out for a minimum of seven weeks. The maximum dose administered was $90 \mathrm{mg} /$ day and the minimum $30 \mathrm{mg} / \mathrm{day}$, with a mean dose of $60 \mathrm{mg} /$ day in six-hourly doses. In one subject the treatment was suspended because of an evident depressive state.

Thus 13 patients in the first group were involved in the CB 154 trial, of whom 10 had also received Madopar treatment previously. Evaluation of the illness was done in the patients on Madopar therapy, in the patients without therapy, and in the patients on CB 154 treatment, so that it was possible to compare the test scores of CB 154 versus no treatment, of Madopar versus no treatment, and of CB 154 versus Madopar.

The second group was formed of a further 12 patients with Parkinson's disease who were on Madopar treatment at the mean daily dose of $700 \mathrm{mg}$. CB 154 was added to the Madopar therapy: for every $10 \mathrm{mg} \mathrm{CB} 154$ given, Madopar was reduced by $125 \mathrm{mg}$ until one half of the effective Madopar dosage was reached, so that these patients were receiving Madopar at the mean dose of $375 \mathrm{mg} /$ day and CB 154 at the mean dose of $25 \mathrm{mg} /$ day. In two cases the addition of CB 154 was suspended because of side effects (postural hypotension) after the first administration. The total number of patients involved in the trial of CB 154 plus Madopar was, therefore, 10. Evaluation of the illness was performed in these

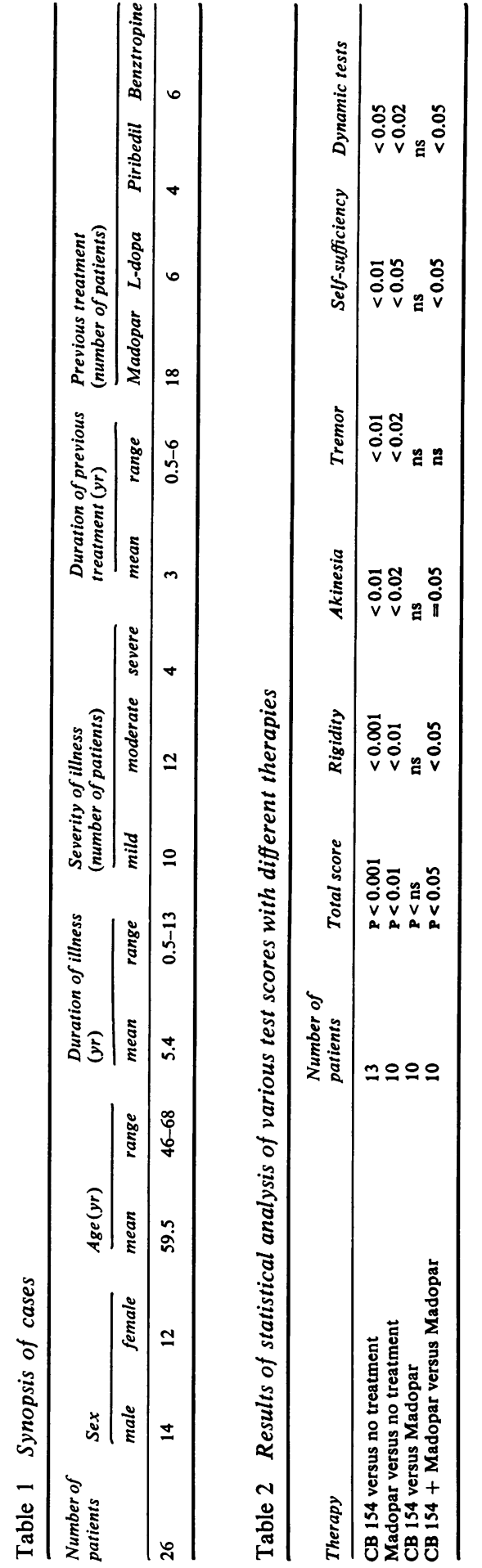


patients during Madopar treatment alone and, during the combined treatment (Madopar plus CB 154).

In evaluating disability we have used the scale proposed by Duvoisin analysing 25 different manifestations of Parkinson's disease, grade 0 being normal and grade 4 characterised by maximum impairment. We evaluated the self-sufficiency of our patients by considering their performance on the following complex daily activities: (1) dressing; (2) buttoning a jacket; (3) tying shoe laces; (4) washing; (5) cutting meat; (6) rising from a chair; (7) movement in bed. These tasks were rated on the scale $0=$ normal execution to $4=$ total inability. We also evaluated the time taken (in seconds) to perform the following simple actions (dynamic tests): (1) rising from a chair, turning round, and returning to the sitting position; (2) walking 11 metres as quickly as possible; and (3) making a 360 degree turn in a supine position in bed. Lastly, all the patients were given an intelligence test (PM38).

These tests were given to the patients once a week for the entire period of the pharmacological trials, and the scores were derived from the mean observation of three different examiners. The best scores of the various tests were used for the statistical evaluation. Statistical analysis was performed using a paired $t$ test (Student's $t$ test).

Careful attention was directed to possible changes of pulse rate, respiration, and standing and lying blood pressure. Side effects such as constipation, nausea, vomiting, asthenia, mouth dryness, psychic disturbance, dyskinesia, paradoxical akinesia, and on-off phenomenon were taken into consideration. Haematological, hepatic, and renal function tests were done before, during, and after our investigation.
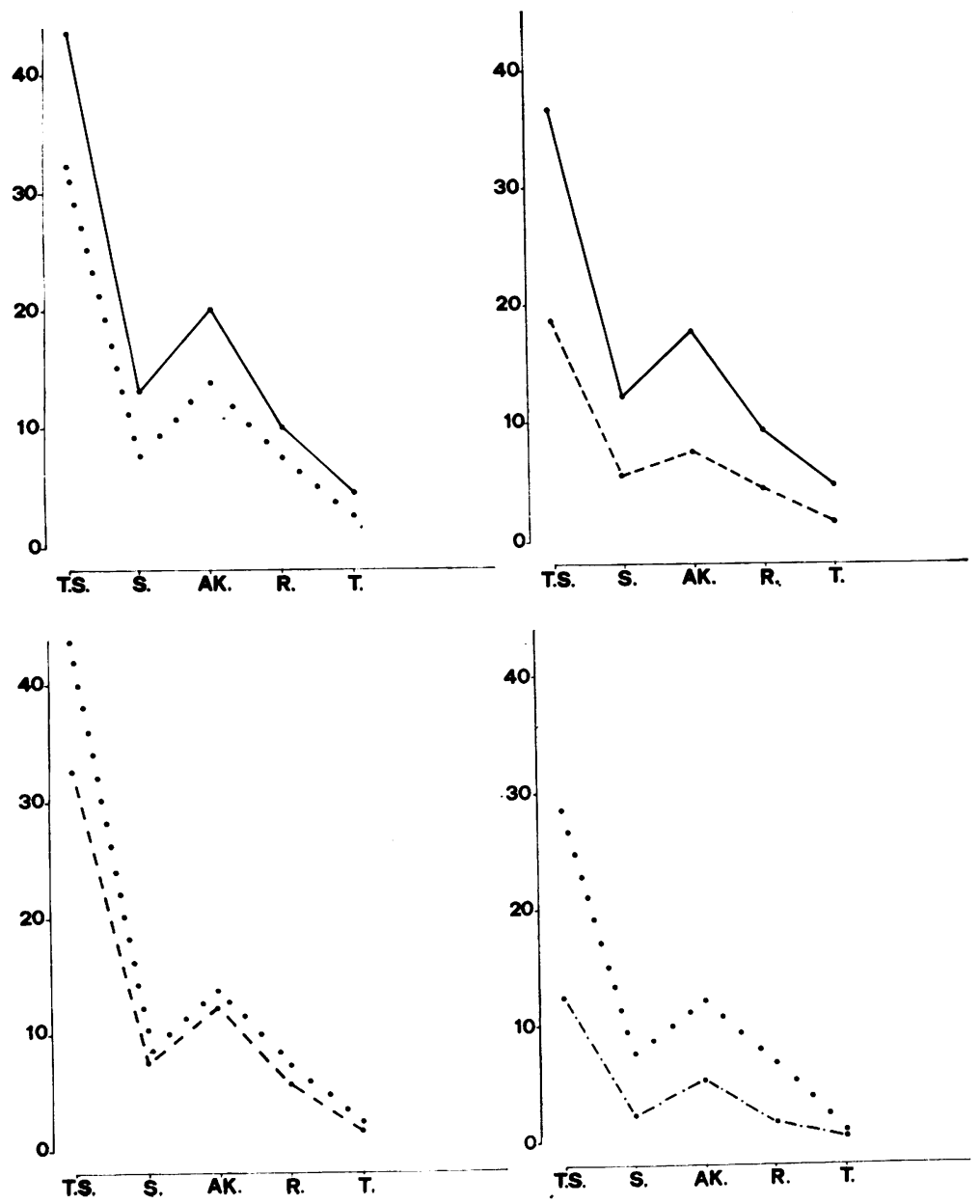

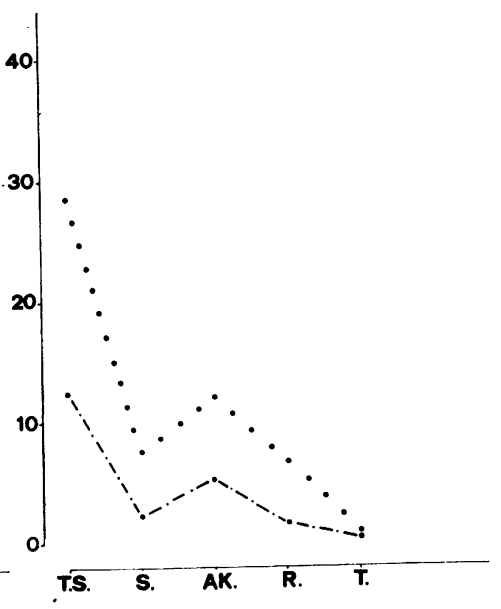

Fig. 1 Graph of the mean score of total disability (T.S.), self-sufficiency $(S$.$) , akinesia$ $(A K$.$) , rigidity (R$.$) , and tremor$ $(T$.) during different therapies and in basal conditions; no therapy; . . . Madopar; - - - CB 154; -_._-_ Madopar and $C B 154$. 


\section{Results}

In the first group of patients we compared the scores obtained in basal conditions to those on CB 154 therapy, in basal conditions to Madopar therapy, and on Madopar to CB 154 therapy. In the second group we compared the scores obtained in Madopar to CB 154 plus Madopar therapy.

Table 2 and Fig. 1 show that a significant improvement of all parameters (total score, hypertonia, akinesia, tremor, self-sufficiency, dynamic tests) was observed after treatment with CB 154 in respect to basal values.

A significant improvement on basal conditions was also evident for Madopar therapy, although the improvement was not as marked as on CB 154 treatment. The impression was that the effect of CB 154 was greater than that of Madopar, but the comparison of the results obtained with CB 154 and those obtained with Madopar was not significant. Comparing CB 154 plus Madopar and Madopar alone, we obtained a much greater improvement on the combined therapy.

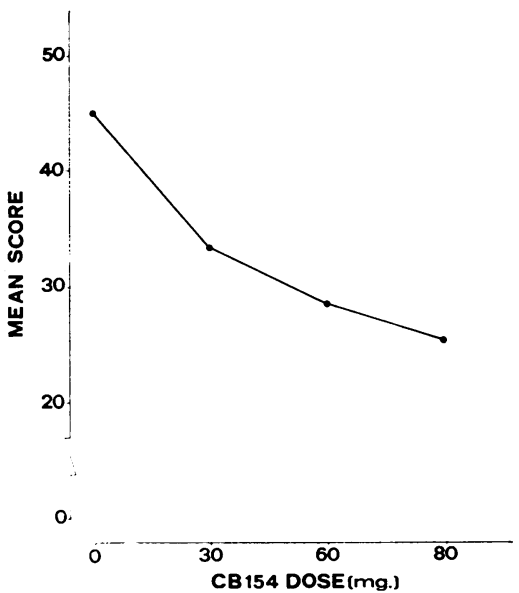

Fig. 2 Reduction in total disability score to bromocriptine 30,60 and $80 \mathrm{mg}$ in 10 patients with Parkinson's disease. Note that the maximal slope of the curve is reached at $30 \mathrm{mg}$ dose level.

We have tried to evaluate the efficacy of CB 154 in respect to dosage. Figure 2 demonstrates that the maximum percentage improvement is reached at the $30 \mathrm{mg}$ dosage level, while higher doses elicit a further improvement but to a lesser degree. The PM 38 psychological test was not significantly modified by the various therapies.

The side effects are shown in Table 3. Gastric disturbances (pain, nausea, vomiting) were most frequent but could be relieved with a simple
Table 3 Side effects

\begin{tabular}{lll}
\hline Effect & \multicolumn{2}{l}{ Treatment } \\
\cline { 2 - 3 } & CB 154 alone & CB 154 + Madopar \\
\hline Gastric disturbances & 8 & 5 \\
Depression & 5 & 2 \\
Postural hypotension & 2 & 3 \\
Dyskinesia & - & 1 \\
On-off phenomenon & - & 1 \\
Confusion & - & 1 \\
\hline
\end{tabular}

antacid treatment. A slight and transitory depressive reaction was observed in three patients with CB 154 and in two with combined treatment. Two other patients on CB 154 treatment were irritable and depressed and the treatment was withdrawn. In both of these patients a similar reaction appeared with subsequent treatment with Madopar, and, therefore, the patients were treated with tricyclic antidepressants which also have some antiparkinsonism effect. In one case the depressive reaction was immediate at the $10 \mathrm{mg}$ dose level of CB 154, and in the other the depression began later at the dose of $30 \mathrm{mg} \mathrm{CB} 154$, when an improvement was already evident. Both patients were affected by an early and very slight form of Parkinson's disease and had not received any treatment before. An increase of hyperkinetic movements was observed in one case, a worsening of mental disturbance (confusion and hallucination) in another. A fall of arterial blood pressure was evident in five cases. Livedo reticularis of limbs appeared in one case.

\section{Discussion}

These results clearly show the effectiveness of bromocriptine (CB 154) in the treatment of Parkinson's disease and are comparable with those previously reported by Kartzinel et al. (1976c) and Parkes et al. (1976). Rigidity and akinesia were significantly reduced, whereas tremor was less effectively alleviated. The appearance of tremor was slight in our patients, and we, therefore, suggest that the statistical analysis might change in a larger population. The scores of self-sufficiency and dynamic tests were also significantly improved (Table 2).

The effectiveness of combined therapy (CB 154 plus Madopar) was significantly higher than that of Madopar alone. There was no significant difference between CB 154 and Madopar treatment although the former therapy seems to be more efficient than the latter (Table 2 and Fig. 1).

A placebo effect must be considered, but we do not attribute the improvement obtained to this in view of the long duration of effectiveness of 
the treatment, the nature of the disease, its organic basis, and the objective evaluation of clinical signs.

The necessity for rather high doses of CB 154 (mean $60 \mathrm{mg} /$ day) has been demonstrated. The mean dose is a little lower than that of Kartzinel et al. (1976a) who, in some arbitrary way, proposed the maximum level of $100 \mathrm{mg} / \mathrm{day}$. These data are at variance with those of Gerlach (1976) who, using smaller dosages, did not find the same improvement in his patients; otherwise our data agree with those of Kartzinel et al. (1976a) who compared the results obtained by CB 154 treatment with those obtained by treatment with $\mathrm{L}$ dopa plus carbidopa (Sinemet).

Taking into consideration the dosage, the effectiveness of CB 154 is maximal at the lower doses (30 $\mathrm{mg}$ a day), while continuing improvement seen at the higher doses $(60-80 \mathrm{mg} /$ day) is less marked (Fig. 2), confirming the previous observations of Kartzinel et al. (1976a).

The addition of CB 154 to Madopar treatment permitted us to reduce the previous treatment by about $50 \%$ in quantity and the number of daily doses.

The side effects of CB 154 are similar to those appearing with L-dopa treatment whether or not associated with a decarboxylase inhibitor. We underline the unusual appearance of a depressive reaction in five patients. Two of the latter had an identical reaction with Madopar treatment, suggesting unpredictable hypersensitivity to dopaminergic stimuli in these cases.

On-off phenomenon or important swings in symptomatology during CB 154 therapy were never observed, and were seen in only one case during CB 154 plus Madopar treatment. This observation, even if restricted to a limited number of patients, seems to confirm the usefulness of bromocriptine in reducing the on-off phenomenon as suggested by Kartzinel and Calne (1976).

In conclusion, on the basis of the results of these trials, we sugggest that CB 154 alone and combined therapy with CB 154 and Madopar, even if further investigations are required, is an important advance in the management of Parkinson's disease.

We thank Sandoz SPA, Milano, for the supply of 2-bromo- $\alpha$-ergocriptine (CB 154). Our thanks are due to Mrs A. Magatti for her secretarial assistance.

\section{References}

Billiter, E., and Flückiger, E. (1971). Evidence for a luteolitic function of prolactin in the intact cyclic rat using 2-br-alpha-ergokryptin (CB 154). Experientia, 27, 464.

Calne, D. B., Teychenne, P. F., Claveria, L. E., Eastman, R., Greenacre, J. K., and Petrie, A. (1974). Bromocriptine in parkinsonism. British Medical Journal, 4, 442-444.

Corrodi, H., Fuxe, K., Hökfelt, T., Libdrink, P., and Ungerstedt, U. (1973). Effect of ergot drugs on central catecholamine neurons: evidence for a stimulation of central dopamine neurons. Journal of Pharmacy and Pharmacology, 25, 409-412.

Flückiger, E., and Wagner, H. R. (1968). 2-br-alphaergokriptin: beeinflussang von fertilitat und laktation bei der ratte. Experientia, 24, 1130-1131.

Gautier, J. C., and Durand, J. P. (1977). Traitement des syndromes parkinsoniens par la bromocriptine. La Nouvelle Presse Médicale, 6, 171-174.

Gerlach, J. (1976). Effect of CB 154 (2-bromo-alphaergocryptine) on paralysis agitans compared with Madopar in a double-blind, cross-over trial. Acta Neurologica Scandinavica, 53, 189-200.

Goldstein, M. A., Battista, A. F., Miyamoto, T. (1975). In Advances in Neurology. Vol 9, pp. 299305. Edited by D. B. Calne, T. N. Chase, and A. Barbeau, Raven Press: New York.

Kartzinel, R., Perlow, M. Teychenne, P., Gielen, A. C., Gillespie, M. M., Sadowsky, D. A., and Calne, D. B. (1976a). Bromocriptine and levodopa (with or without carbidopa) in parkinsonism. Lancet, 2, 272-275.

Kartzinel, R., Shoulson, I., and Calne, D. B. (1976b). Studies with bromocriptine. III. Concomitant administration of caffeine to patients with icliopathic parkinsonism. Neurology (Minneapolis), 26, 741743.

Kartzinel, R., Shoulson, I., and Calne, D. B. (1976c). Studies with bromocriptine. Part 2. Double-blind comparison with levodopa in idiopathic parkinsonism. Neurology (Minneapolis), 26, 511-513.

Kartzinel, R., and Calne, D. B. (1976). Studies with bromocriptine. Part I. 'On-off' phenomena. Neurology, (Minneapolis), 26, 508-510.

Lieberman, A., Miyamoto, T., Battista, A., and Goldstein, M. (1975). Studies on the antiparkinsonian efficacy of lergotrile. Neurology (Minneapolis), 25, 459-462.

Lieberman, A., Zolfaghari, M., Boal, D., Massouri, H., Vogel, B., Battista, A., Fuxe, K., and Goldstein, M. (1976a). The antiparkinsonian efficacy of bromocriptine. Neurology (Minneapolis), 26, 405-409.

Lieberman, A.. Kupersmith, M., Estey, E., and Goldstein. M. (1976b). Treatment of Parkinson's disease with bromocriptine. New England Journal of Medicine, 295, 1400-1404.

Miyamoto, T., Battista, A., Goldstein, M., and Fuxe, K. (1974). Long-lasting anti-tremor activity incluced by 2-Br-alpha-ergocryptine in monkeys. Journal of Pharmacy and Pharmacology. 26, 452-454.

Parke;. J. D.. Marsden. C. D.. Donaldson, I.. GaleaDebono, A.. Walters, J., Kennedy, G., and Asselman, P. (1976). Bromocriptine treatment in Parkinson's disease. Journal of Neurology, Neurosurgery, and Psychiatry, 30, 184-193. 